\title{
Importância atribuída ao sexo por idosos do município de Porto Alegre e associação com a autopercepção de saúde e o sentimento de felicidade
}

Importance given to sex by the elderly in the city of Porto Alegre and association

between self-health perception and the feeling of happiness

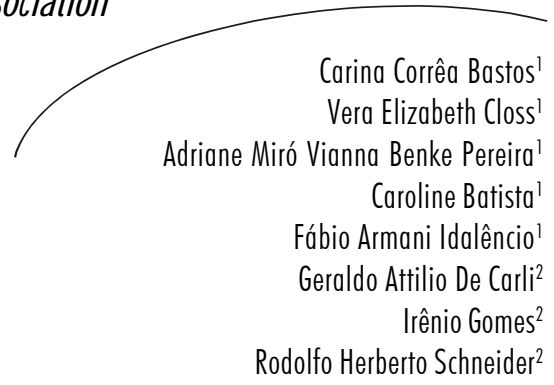

Resumo

Introdução: $\mathrm{O}$ envelhecimento da população tem ocasionado transformações na vida cotidiana dos idosos, revelando a necessidade de uma constante revisão das necessidades deste segmento populacional, dentre as quais aquelas relativas às manifestações da sexualidade. Aspectos relacionados à sexualidade dos idosos brasileiros ainda são pouco conhecidos, e envolvem mitos e preconceitos a serem superados. Objetivos: Verificar a importância atribuída ao sexo por idosos da cidade de Porto Alegre-RS, Brasil, e analisar a associação com a autopercepção de saúde e sentimento de felicidade. Métodos: Pesquisa realizada a partir da análise do banco de dados do Estudo Multidimensional dos Idosos de Porto Alegre (EMIPOA), com 1.078 indivíduos de 60 anos de idade ou mais. Resultados: Participaram do estudo 938 idosos, com idades entre 60 e 95 anos, dos quais 542 $(57,8 \%)$ consideraram o sexo muito importante ou importante. Houve diferença significativa $(\mathrm{p}<0,001)$ na importância dada ao sexo por homens e mulheres, e não se observou associação significativa entre importância atribuída ao sexo e sentimento de felicidade $(\mathrm{p}=0,299)$. Conclusão: $\mathrm{O}$ sexo é considerado importante por grande parte dos idosos, tema que poderia ser abordado mais amplamente por profissionais da saúde.

\section{Abstract}

Introduction: Ageing has brought many modifications into the life of elderly people revealing the necessity of a close assistance to their needs and among them there is the very special one focusing their sexual activity. Sexual aspects about brasilian elderly are still little known, involving myths and prejudice to be overcome. Objective: verify the degree of importance given to sex by the elderly in Porto Alegre city, RS, Brazil and analyze the association between self-health perception and the feeling of happiness. Methods: Research was done through the analysis

\footnotetext{
Programa de Pós-graduação em Gerontologia Biomédica. Pontifícia Universidade Católica do Rio Grande do Sul - PUCRS. Porto Alegre, RS, Brasil.

2 Instituto de Geriatria e Gerontologia. Pontifícia Universidade Católica do Rio Grande do Sul PUCRS. Porto Alegre, RS, Brasil.
}

Palavras-chave: Sexualidade. Sexo. Idosos. Saúde. Felicidade. 
of the Multidimensional Study of the Elderly Database of Porto Alegre (EMIPOA) involving 1,078 individuals aged 60 and over. Results: Study conducted with 938 elderly people aged $60-95$ years; $542(57,8 \%)$ considered sex very important and important. There was a significant difference $(\mathrm{p}<0,001)$ in the importance given to sex by men and women and no significant association between the importance given to sex and the feeling of happiness $(p=0,299)$ was observed. Conclusion: Sex is considered important by a great number of old people, that is open to a more profound study for sure.
Key words: Sexuality. Sex. Elderly. Health. Happiness.

\section{INTRODUÇÃO}

Pesquisas e estudos populacionais vêm informando à sociedade que o envelhecimento da população tem proporções significativas. ${ }^{1}$ No Brasil, a literatura mostra um aumento percentual de idosos, que somam $11,1 \%$ da população, perfazendo um total de 21 milhões de pessoas. ${ }^{2}$

O Instituto Brasileiro de Geografia e Estatística (IBGE) considera idosos os indivíduos com 60 anos ou mais, ${ }^{1}$ mesmo limite de idade considerado pela Organização Mundial da Saúde (OMS) para os países em desenvolvimento. ${ }^{1}$ As projeções indicam que a população idosa aumentará, continuamente, aproximando-se de $20 \%$ da população total brasileira em $2050 .^{2}$

O envelhecimento da população brasileira é reflexo do aumento da expectativa de vida, devido ao avanço no campo da saúde e à queda nas taxas de fecundidade e mortalidade. Isso vem ocasionando mudanças na estrutura etária, com diminuição relativa da população mais jovem e aumento proporcional dos idosos. ${ }^{3}$

Este fenômeno mundial tem ocasionado transformações na vida cotidiana dos idosos em diferentes aspectos e várias mudanças já estão sendo sentidas de forma bastante concreta, revelando a necessidade de constante revisão das necessidades deste segmento populacional. ${ }^{1}$ Entre essas necessidades encontram-se, também, as relativas às manifestações da sexualidade. ${ }^{4}$

A forma de expressão da sexualidade e o desejo por momentos de intimidade são complexos, já que sofrem influência de fatores biopsicossociais, econômicos, culturais, religiosos e espirituais. A noção de saúde sexual, como a de saúde física, não é simplesmente a ausência de disfunção ou doença sexual, mas um estado de bem-estar sexual que inclui aproximação positiva de um relacionamento e antecipação de uma experiência prazerosa sem medo, vergonha, violência ou coação. $^{5}$

$\mathrm{Na}$ cultura brasileira, vários mitos e atitudes sociais são atribuídos às pessoas idosas, dificultando a manifestação da sexualidade em suas vidas. ${ }^{6}$ Segundo a psicogerontologia, a experiência subjetiva do envelhecimento é amplamente influenciada pela ideologia cultural. A vivência subjetiva é marcada pela inevitabilidade das modificações corporais e das competências físicas, pelas modificações em nível dos recursos cognitivos e adaptativos, pelas alterações de papéis e da posição nas hierarquias sociais, assim como pelo impacto negativo de atitudes e estereótipos relativos ao envelhecimento. A crença na progressiva e generalizada incompetência, assim como na impotência sexual dos idosos, é parte intrínseca desses estereótipos. Acuados entre as múltiplas exigências adaptativas que as alterações do envelhecimento comportam, os indivíduos enfrentam dificuldades para preservar a identidade pessoal e a integridade de alguns papéis e funções, sobretudo aqueles relativos à sexualidade que a sociedade atentamente vigia e sanciona?

A sexualidade na velhice é vista como tabu para os que têm idade mais avançada, pois a sociedade ainda concebe que apenas aos mais novos é dada a possibilidade de manifestar sua sexualidade, 
relegando o idoso à abstinência sexual. Assim, pode até existir um desejo de ir ao encontro do outro por parte dos idosos, mas não há motivação suficiente, pois acreditam que, se o fizerem, serão estigmatizados como pervertidos a partir dos ditames que lhes são impostos. ${ }^{8,9}$

No entanto, a sexualidade faz parte da vida dos seres humanos, estando presente em todas as fases do desenvolvimento, desde o nascimento até a morte. A função sexual continua por toda a vida, mesmo no envelhecimento, embora a sexualidade sob a perspectiva da vivência e o envelhecimento sejam vistos como incompatíveis. ${ }^{4}$

Há escassez de relatos sobre o significado do relacionamento sexual para idosos, embora os poucos estudos publicados mostrem que $\mathrm{o}$ interesse e atividade sexuais persistam no envelhecimento. ${ }^{10} \mathrm{Um}$ dos primeiros estudos qualitativos a envolver idosos, diretamente na discussão sobre sexualidade e saúde sexual, tem mudado este paradigma, por identificar que o sexo representa importante aspecto para a qualidade de vida no envelhecimento. ${ }^{11}$

Nesse contexto, será apresentada uma análise de dados relativos à sexualidade dos participantes do Estudo Multidimensional dos Idosos de Porto Alegre (EMIPOA), partindo da hipótese de que esta seja uma questão que permanece importante com o avançar da idade.

\section{METODOLOGIA}

Trata-se de estudo transversal, de base populacional, com idosos (60 anos ou mais) do município de Porto Alegre, Rio Grande do Sul, Brasil. Este estudo fez parte do Estudo Multidimensional dos Idosos de Porto Alegre (EMIPOA), realizado pelo Instituto de Geriatria e Gerontologia (IGG) - PUCRS, no período de janeiro a setembro de 2006.

O tamanho da amostra foi calculado para 1.092 idosos, baseando-se no número de indivíduos avaliados em estudo anterior (1995), que estudou $0,69 \%$ da população de idosos, de acordo com as estimativas de variação populacional para 2005, para cada bairro da cidade de Porto Alegre, atualizado pelo Instituto Brasileiro de Geografia e Estatística (IBGE). Os entrevistados foram recrutados de forma estratificada e proporcional por região censitária, com seleção aleatória do primeiro domicílio de cada região e sistematizada dos demais, sendo sorteado um idoso por domicílio. Foram excluídos idosos acamados, com impossibilidade de locomoção, com déficit cognitivo ou transtorno psiquiátrico severo e os que se recusaram a participar da pesquisa. Aplicou-se um questionário com dados socioeconômicos e de saúde, em 1.078 idosos, por profissionais treinados do Serviço Social da PUCRS, nas residências escolhidas. Dos dados coletados, relacionaram-se questões sobre sexo, estado civil, faixa etária, autopercepção de saúde, importância do sexo, sentimento de felicidade e formas de manifestação da sexualidade. A questão abrangendo opinião sobre o sexo foi reagrupada da seguinte forma: a opção de resposta "muito importante" permaneceu; as opções "natural”, "necessário" e "igual às outras idades" foram agrupadas no termo "Importante"; as opções "menos intenso", "desnecessário" e "indiferente" foram agrupadas no termo "Pouco Importante". Já na questão que abrangeu autopercepção de saúde, as opções de resposta, que eram "ótima”, "boa", "regular", "má" e "péssima”, foram reorganizadas da seguinte forma: boa (ótima ou boa), regular (regular) e ruim (má ou péssima).

Os dados foram organizados em um banco de dados e, posteriormente, analisados com o programa estatístico SPSS, versão 11 . Os testes estatísticos utilizados foram o teste do quiquadrado de Pearson para variáveis categóricas não ordinais, e o teste de tendência linear do quiquadrado para as variáveis categóricas ordinais. Em atendimento à Resolução 196/96, do Conselho Nacional de Saúde, ${ }^{12}$ o projeto foi submetido ao Comitê de Ética em Pesquisa da PUCRS, tendo sido aprovado sob o ${ }^{\circ} 31 / 2006$, e todos os idosos participantes do estudo assinaram o Termo de Consentimento Livre e Esclarecido. 


\section{RESULTADOS}

Dos 1.078 idosos entrevistados no EMIPOA, 938 (87\%) responderam à questão: éfeliz na maior parte do tempo? Estes foram considerados para o estudo e os demais excluídos da amostra. Dos incluídos, 527 (56,2\%) deram parecer quanto à importância atribuída ao sexo, e $411(43,8 \%)$ se omitiram. A amostra foi constituída de 661 (70,5\%) mulheres e, em relação ao estado civil, $359(38,3 \%)$ eram viúvos e $357(38,1 \%)$, casados. A idade variou de 60 a 95 anos, sendo que 420 $(44,8 \%)$ dos idosos se encontravam na faixa etária de 60 a 69 anos.

Noventa por cento dos idosos relataram felicidade na maior parte do tempo. Não foi observada diferença significativa nesta frequência em relação ao sexo e a faixa etária (tabela1). Foi observada tendência ao sentimento de felicidade ser maior entre os idosos casados $(P=0,097)$. Entre as manifestações da sexualidade, "carinhos e toques" e "atenções especiais" foram as opções mais escolhidas (83,6\%), não havendo associação entre esta variável e o sentimento de felicidade. $\mathrm{Na}$ relação entre sentimento de felicidade e autopercepção de saúde, 94,1\% dos idosos que relataram boa saúde sentiam-se felizes na maior parte do tempo, enquanto que, entre os que relataram saúde ruim, o percentual desse sentimento se reduziu para $70,5 \%(P<0,001)$.

Dos indivíduos estudados, 542 (57,8\%) consideraram o sexo "muito importante" ou "importante" (tabela 2). O sexo foi considerado muito importante por $16,6 \%$ dos homens e $7,0 \%$ das mulheres, e pouco importante por $30,3 \%$ dos homens e $47,2 \%$ das mulheres, sendo esta diferença entre os sexos estatisticamente significativa $(P<0,001)$. Não se observou associação significativa entre o estado civil e a importância atribuída ao sexo. Observou-se que quanto maior a idade, menor importância é atribuída ao sexo $(P<0,001)$. Em relação à manifestação da sexualidade, quando esta é por "atenções especiais" a importância do sexo é menor, sendo maior quando é por "relações sexuais" $(P<0,001)$.

Houve a tendência de se atribuir maior importância ao sexo por aqueles que possuem melhor percepção de saúde $(P=0,066)$. No presente estudo, não se observou associação entre o sentimento de felicidade e a importância atribuída ao sexo $(P=0,299)$. 
Tabela 1 - Frequência do relato de sentimento de felicidade na maior parte do tempo, de acordo com as características demográficas, a forma de manifestação da sexualidade e a autopercepção de saúde, em 938 idosos (60 anos ou mais) residentes no município de Porto Alegre, RS.

\begin{tabular}{lccc}
\hline Variável & População & Relato de felicidade & \multirow{2}{*}{ N (\%) } \\
\hline Sexo & $277(29,5)$ & 91,0 & $0,511^{*}$ \\
$\quad$ Masculino & $661(70,5)$ & 89,6 & \\
Feminino & & & \\
Estado civil & $139(14,8)$ & 89,9 & $0,097^{*}$ \\
Solteiro & $357(38,1)$ & 92,2 & \\
Casado & $359(38,3)$ & 89,4 & \\
Viúvo & $83(8,8)$ & 83,1 & $0,659^{\$}$ \\
Separado & & & \\
Idade & $420(44,8)$ & 89,0 & \\
60-69 anos & $356(37,9)$ & 90,4 & \\
70-79 anos & $162(17,3)$ & 91,4 & $0,964^{*}$ \\
80 anos ou mais & & & \\
Manifestação da sexualidade & $220(41,8)$ & 90,5 & \\
Atenções especiais & $221(41,9)$ & 90,5 & \\
Carinhos e toques & $86(16,3)$ & 89,5 & \\
Relações sexuais & & $90,01^{\$}$ \\
Autopercepção de saúde & $459(48,9)$ & 94,1 & \\
Saúde boa & $401(42,8)$ & 89,0 & \\
Saúde regular & $78(8,3)$ & 70,5 & \\
Saúde ruim & $938(100)$ & 90,0 & \\
\hline TOTAL & & & \\
\hline
\end{tabular}

* Valor de P calculado pelo teste do qui-quadrado de Pearson.

$\$$ Valor de $P$ calculado pelo teste de tendência linear do qui-quadrado. 
Tabela 2 - Distribuição do grau de importância atribuído ao sexo, de acordo com as características demográficas, a forma de manifestação da sexualidade, a autopercepção de saúde e a sensação de felicidade, em 938 idosos (60 anos ou mais) residentes no município de Porto Alegre, RS.

\begin{tabular}{|c|c|c|c|c|}
\hline \multirow[b]{2}{*}{ Variáveis } & \multicolumn{3}{|c|}{ Importância atribuída ao sexo } & \multirow[b]{2}{*}{$P$} \\
\hline & $\begin{array}{c}\text { Muito } \\
\text { importante } \\
\%\end{array}$ & $\begin{array}{c}\text { Importante } \\
\%\end{array}$ & $\begin{array}{c}\text { Pouco } \\
\text { importante } \\
\%\end{array}$ & \\
\hline \multicolumn{5}{|l|}{ Sexo } \\
\hline Masculino & 16,6 & 53,1 & 30,3 & \multirow{2}{*}{$<0,001 *$} \\
\hline Feminino & 7,0 & 45,8 & 47,2 & \\
\hline \multicolumn{5}{|l|}{ Estado civil } \\
\hline Solteiro & 10,8 & 41,7 & 47,5 & \multirow{4}{*}{$0,116 *$} \\
\hline Casado & 11,5 & 51,5 & 37,0 & \\
\hline Viúvo & 8,1 & 45,7 & 46,2 & \\
\hline Separado & 8,4 & 53,0 & 38,6 & \\
\hline \multicolumn{5}{|l|}{ Idade } \\
\hline 60-69 anos & 11,9 & 55,0 & 33,1 & \multirow{3}{*}{$<0,001^{\$}$} \\
\hline 70-79 anos & 9,8 & 43,3 & 46,9 & \\
\hline 80 anos ou mais & 4,3 & 40,1 & 55,6 & \\
\hline \multicolumn{5}{|c|}{ Manifestação da sexualidade } \\
\hline Atenções especiais & 7,7 & 51,8 & 40,5 & \multirow{3}{*}{$<0,001^{*}$} \\
\hline Carinhos e toques & 16,7 & 52,5 & 30,8 & \\
\hline Relações sexuais & 15,1 & 69,8 & 15,1 & \\
\hline \multicolumn{5}{|c|}{ Autopercepção de saúde } \\
\hline Saúde boa & 11,3 & 51,0 & 37,7 & \multirow{3}{*}{$0,066^{\$}$} \\
\hline Saúde regular & 8,2 & 45,9 & 45,9 & \\
\hline Saúde ruim & 9,0 & 41,0 & 50,0 & \\
\hline \multicolumn{5}{|l|}{ Sensação de felicidade } \\
\hline $\operatorname{Sim}$ & 11,7 & 40,4 & 47,9 & \multirow{2}{*}{$0,299 *$} \\
\hline Não & 9,6 & 48,8 & 41,6 & \\
\hline TOTAL & 9,8 & 48,0 & 42,2 & \\
\hline
\end{tabular}

* Valor de $P$ calculado pelo teste do qui-quadrado de Pearson.

s Valor de $P$ calculado pelo teste de tendência linear do qui-quadrado.

\section{DISCUSSÃO}

A sexualidade humana é, ainda hoje, uma área de investigação pouco explorada, e o número de pesquisas sobre o assunto associado ao envelhecimento é incipiente. Nos últimos dez anos, grande número de estudos tem investigado a prevalência de problemas sexuais entre os idosos, com foco mais voltado para as alterações sexuais masculinas, como a disfunção erétil e a ejaculação precoce. ${ }^{10}$ Grande parte das pesquisas aborda a perspectiva biológica ou médica da sexualidade humana presumindo que, conforme as pessoas envelhecem, mudanças físicas, hormonais e doenças crônicas reduzem ou eliminam a libido e o comportamento sexual. ${ }^{13}$ Essas alterações 
físicas e hormonais podem estar relacionadas à autopercepção de saúde nos idosos. ${ }^{14}$

No Brasil, os estudos que abordam sexualidade são ainda mais escassos. O Ministério da Saúde, em sua publicação Cadernos de Atenção Básica Envelhecimento e Saúde da Pessoa Idosa (n. 19), enfatiza a necessidade de se lidar com a questão sexual desta população devido não só ao envelhecimento populacional e crescimento das doenças transmissíveis (Aids, em especial) entre indivíduos com mais de 60 anos, mas também por se tratar de elemento importante para a qualidade de vida deste grupo etário. ${ }^{15}$

No Reino Unido, em estudo envolvendo 21 homens e 23 mulheres com idade entre 50 e 92 anos, avaliando qualitativa e quantitativamente os dados sobre a prioridade do sexo na idade avançada, observou-se que todos os indivíduos que tinham um parceiro sexual atribuíram ao sexo certa importância. ${ }^{11}$ Fato semelhante foi observado no presente estudo, onde os casados atribuíram maior importância ao sexo, embora este dado não tenha mostrado associação significativa.

A importância conferida ao sexo diminui à medida que aumenta a presença de problemas relacionados à saúde. ${ }^{11}$ No presente estudo, observou-se que indivíduos que possuem melhor percepção de saúde tendem a atribuir maior importância ao sexo. Segundo Gott et al., o sexo pode deixar de ser importante quando há intercorrências como a morte do cônjuge ou doença grave. ${ }^{6}$

Além das mudanças físicas naturais durante o processo de envelhecimento, os indivíduos estão mais susceptíveis a problemas de saúde como diabetes mellitus e hipertensão. Tais doenças podem impactar negativamente sobre a prática sexual, já que a má circulação causada por elas influencia tanto na libido sexual quanto na ereção. Entretanto, desfrutar de boa saúde não consiste em impedimento para que a pessoa mantenha atividade sexual, podendo esta sofrer modificações ao longo da vida. ${ }^{16}$ A terapia farmacológica utilizada em idosos também pode contribuir para o surgimento de alterações sexuais. ${ }^{17}$
Ainda em relação à importância atribuída ao sexo e percepção de saúde, Freedman afirma que a forma que o idoso tem a respeito da sua imagem corporal durante o envelhecimento exerce influência no seu comportamento e na sua autoestima. ${ }^{18}$

Nicolosi et al., em estudo multicêntrico realizado em 29 países entre 2001 e 2002, envolvendo 4.977 homens e 5.023 mulheres com idade entre 40 a 80 anos, observaram que $18 \%$ da população mostraram perda de interesse pelo sexo; $17 \%$ dos homens e $23 \%$ das mulheres afirmaram que "pessoas idosas não desejam sexo por mais tempo. Já no presente estudo, observou-se que $30,3 \%$ dos homens e $47,2 \%$ das mulheres consideraram o sexo pouco importante, sendo este percentual maior quando comparado ao estudo realizado por Nicolosi et al. ${ }^{19}$

Os resultados aqui obtidos são concordantes com os obtidos por Lindau et al. em 2007 sobre a prevalência de atividade sexual em amostra de 3.005 indivíduos (1.550 mulheres e 1.455 homens) nos Estados Unidos, em que 35\% das mulheres consideraram o sexo como sendo "não tão importante" quando comparado à opinião de $13 \%$ dos homens. ${ }^{20}$ Esse achado pode ser atribuído a questões culturais e de educação, pois os diferentes papéis de gêneros ficaram mais evidentes, sobretudo na relação da moral com a questão sexual. Assim, a moralidade conduzia a experiências sexuais masculinas, enquanto procurava restringir a sexualidade feminina. ${ }^{21}$

Embora ocorram modificações no corpo do homem e da mulher durante o processo normal de envelhecimento, as mulheres percebem as alterações sexuais advindas da idade com menos impacto no exercício sexual. ${ }^{22}$ Por ser uma função fisiológica, a atividade sexual é comumente confundida com o ato sexual, não sendo observadas outras formas de manifestação da sexualidade, como demonstrações de carinho, afeto, companheirismo e ternura. ${ }^{23}$

No entanto, em pesquisa realizada com idosos que frequentavam a Universidade da Terceira Idade na Universidade Metodista de Piracicaba em 1992, 
verificou-se que eles sentiam necessidade de atrair o sexo oposto, mas esta atração está mais ligada à necessidade de querer conviver com outra pessoa do que propriamente com o ato sexual. ${ }^{24}$ Desta forma, fica demonstrada uma manifestação da sexualidade mais relacionada ao campo sentimental.

Em relação ao aspecto físico da sexualidade, como o toque, o abraço ou as carícias, os viúvos relataram sentir falta dessa proximidade, ${ }^{25}$ fatores que, no presente estudo, têm relação com o sentimento de felicidade. Neste aspecto, observouse que $41,7 \%$ dos idosos diziam manifestar sua sexualidade através de atenções especiais, $41,9 \%$, através de carinhos e toques e $16,3 \%$, através de relações sexuais.

Estudo brasileiro realizado por Silva acerca da manifestação da sexualidade, envolvendo 36 indivíduos, em sua maioria mulheres, com média de idade de 70 a 75 anos, participantes do Grupo de Educação e Saúde (GES) da Divisão de Medicina de Reabilitação da Faculdade de Medicina da Universidade de São Paulo (DMRHCFMUSP), focou as alterações na função sexual dos idosos e as expectativas dos mesmos com relação à sexualidade. Silva concluiu que a forma de estímulo mais citada foi a de carícias (56\%), seguida por carícias e beijos (22\%). ${ }^{26}$ No presente estudo, observou-se que $41,9 \%$ dos idosos manifestaram preferência por carinhos e toques como forma de demonstração da sexualidade.

Pesquisas acerca do desejo sexual ainda são conflitantes. Há estudos na literatura indicando que o desejo sexual decresce com a idade, enquanto outros referem não ocorrer mudança na sexualidade com o avançar da idade, desde que a saúde permaneça preservada. ${ }^{13}$

Além das dificuldades inerentes à abordagem de assuntos envoltos pelo tema sexualidade, podem ser apontadas limitações de caráter metodológico neste estudo. Por ser um estudo retrospectivo, ressalta-se a impossibilidade de incluir variáveis que poderiam auxiliar na análise dos resultados e as perdas decorrentes de dados incompletos nas respostas dos idosos. Existe também o risco de vieses ou distorções por parte do investigador ao questionar retrospectivamente, baseado na memória do idoso. Independentemente dessas limitações, o presente estudo concluiu que o sexo não é fator determinante para o sentimento de felicidade dos idosos, embora o sexo tenha sido percebido de forma positiva pela maior parte da população avaliada, mostrando não haver estagnação do desejo sexual com o envelhecimento.

\section{CONCLUSÃO}

Embora o processo de envelhecimento passe por mudanças físicas, bioquímicas e funcionais que podem exercer influência no sentido de reduzir a atividade sexual, muitos idosos ainda apresentam importante interesse sexual aos 70, 80 e mesmo 90 anos de idade. ${ }^{11}$ No entanto, esse interesse pode ser manifestado de outras formas, como carinhos, toques e companheirismo.

Sendo os idosos um percentual expressivo de usuários do sistema de saúde, é importante que os profissionais de saúde questionem sobre a vida sexual desses indivíduos, para que se sintam confiantes e possam receber orientação, vislumbrando melhor qualidade de vida. Vale salientar que os profissionais de saúde não têm ainda, na rotina diária, o hábito de questionar aspectos ligados à sexualidade dos idosos. ${ }^{27}$

Estudos abordando o tema sexualidade ainda são incipientes na literatura, com poucos autores que abordam esta temática, inclusive no Brasil. Dessa forma, investigações se fazem necessárias, visto que ainda existem preconceitos em torno desse assunto, tanto por parte dos profissionais da saúde e pela sociedade quanto pelos próprios idosos. 


\section{REFERÊNCIAS}

1. Instituto Brasileiro de Geografia e Estatística (IBGE). Síntese de Indicadores Sociais . Rio de Janeiro : IBGE; 2009. Disponível em: http:// www.ibge.gov.br/home/estatistica/ populacao/condicaodevida/ indicadoresminimos/sinteseindicsociais2009/ indic_sociais2009.pd

2. Pereira AMVB, Schneider RH, Schwanke CHA. Geriatria, uma especialidade centenária. Scientia Medica 2009; 19(4):154-161.

3. Pavarini SCI, et al. A arte de cuidar do idoso: gerontologia como profissão? Text \& Context $2005 ; 14: 398-402$.

4. Almeida T, Lourenço ML. Envelhecimento, amor e sexualidade: utopia ou realidade? Rev Bras de Geriatr Gerontol 2007;10:101-13.

5. Rheaume C, Mitty E. Sexuality and Intimacy in older adults. Geriatric Nursing 2008; 29:342-49.

6. Gott M, Hinchliff S, Galena E. General practioners attitudes to discussing sexual health issues with older people. Soc Sci Med 2004; 58:2009-13.

7. Vasconcelos $\mathrm{D}$, et al. A sexualidade no processo do envelhecimento: novas perspectivas. Estud Psicol 2004; 9(3):413-419.

8. Lowndes L. Como fazer qualquer pessoa se apaixonar por você. 9 ed. Rio de Janeiro: Record; 2002.

9. Biddulph S.Por que escolhi você? São Paulo: Fundamento; 2003.

10. Moreira Jr, et al. Prevalence of sexual problems and related help-seeking behaviors among mature adults in Brazil: data from the global study of sexual attitudes and behaviors. Sao Paulo Med J 2005; 123:234-41.

11. Gott M, Hinchliff S. How important is sex in later life? The views of older people. Soc Sci Med 2003; 56:1617-28.

12. Ministério da Saúde (Brasil). Conselho Nacional de Saúde. Resolução n. 196, de 5 a 10 de outubro de 1996. Brasília: Ministério da Saúde; 1996.
13. De Lamater JD. Sexual desire in later life. University of Michigan. J Sex Res. 2005; 42:138-49.

14. Negreiros MA. Prevenção do declínio cognitivo. In: Negreiros TCGM, organizador. A nova velhice: uma visão multidisciplinar. Rio de Janeiro: Revinter; 2002.

15. Ministério da Saúde (Brasil). Envelhecimento saúde da pessoa idosa. Saúde da Família. Cadernos de Atenção Básica no 19 Brasília: MS; 2006.

16. Almeida AB. Climatério. Porto Alegre: Artes Médicas; 1993.

17. Risman A. Sexualidade e o homem idoso. Arq Geriatr Gerontol 1996; 10(1):123-4.

18. Freedman R. Meu corpo, meu espelho. Rio de Janeiro: Rosa dos Tempos; 1994.

19. Nicolosi A, et al. Sexual behaviour, sexual dysfunctions and related help seeking patterns in middle-aged and elderly Europeans: the global study of sexual attitudes and behaviors. World J Urol 2006; 24:423-28.

20. Lindau ST, et al. A Study of sexuality and health among older adults in the United States. The N Engl J Med 2007;357:762-74.

21. Marques B. Íntimo e especial. Rev Vida e Saúde 2002; 64(3):48-9.

22. Patrizzi LJ. Um certo clima. Rev Vida Saúde 2004;66(9):18-20.

23. Dantas JMR, et al. Lazer e sexualidade no envelhecer. Estud Psicol. 2004; 9:413-19.

24. Simões R. Corporeidade e terceira idade: a marginalização do corpo do idoso. Piracicaba:Unimep; 1998.

25. Argimon IL, Vitola JC. Estratégias para facilitar a convivência. In: Dornelles B, Costa GJC. Investindo o envelhecimento saudável. Porto Alegre:EDIPUCRS; 2003.

26. Silva RMO. A sexualidade no envelhecer: um estudo com idosos em reabilitação. Acta fisiátrica 2003; 10:107-12.

27. Gradim CVC, Sousa AMM, Lobo JM. A prática sexual e o envelhecimento. Cogitare Enferm 2007;12:204-13. 
